GANIT J. Bangladesh Math. Soc. (ISSN 1606-3694) 37 (2017) 161-174

\title{
NUMERICAL SOLUTIONS OF SYSTEM OF SECOND ORDER BOUNDARY VALUE PROBLEMS USING GALERKIN METHOD
}

\author{
Mahua Jahan Rupa' ${ }^{1}$ and Md. Shafiqul Islam² \\ ${ }^{1}$ Department of Mathematics, University of Barisal, Barisal-8200, Bangladesh \\ ${ }^{2}$ Department of Applied Mathematics, University of Dhaka, Dhaka-1000, Bangladesh \\ ${ }^{2}$ Corresponding author: email: mdshafiqul@du.ac.bd
}

Received 26.07.2017 Accepted 26.10.2017

\begin{abstract}
In this paper we derive the formulation of one dimensional linear and nonlinear system of second order boundary value problems (BVPs) for the pair of functions using Galerkin weighted residual method. Here we use Bernstein and Legendre polynomials as basis functions. The proposed method is tested on several examples and reasonable accuracy is found. Finally, the approximate solutions are compared with the exact solutions and also with the solutions of the existing methods.
\end{abstract}

Keywords: Galerkin method, second order linear and nonlinear BVP, Bernstein and Legendre polynomials.

\section{Introduction}

Ordinary differential systems have been focused in many studies due to their frequent appearance in various applications in physics, engineering, biology and other fields. Wazwaz [1] applied the Adomian decomposition method to solve singular initial value problems in the second order ordinary differential equations, Ramos [2] proposed linearization techniques for solving singular initial value problems (IVPs) of ordinary differential equations, and there are other papers [7 - 9] for solving second order IVPs. However, many classical numerical methods used to solve secondorder IVPs that cannot be applied to second order BVPs. For a nonlinear system of second order BVPs [3], there are few valid methods to obtain the numerical solutions. Many authors [10, 11] discussed the existence of solutions to second order systems, including the approximation of solutions via finite difference method. Lu [4] proposed the variational iteration method for solving a nonlinear system of second order BVPs. Since piecewise polynomials can be differentiated and integrated easily and can be approximated to any function of any accuracy desired. Hence Bernstein polynomials have been used by many authors. Very recently, Bhatti and Bracken [5] used Bernstein polynomials for solving two point second order BVP, but it is limited only to first order nonlinear IVP. Besides spline functions and Bernstein polynomials, there are another type of piecewise continuous polynomials, namely Legendre polynomials [6]. 
Therefore, the purpose of this paper is devoted to use two kinds of piecewise polynomials: Bernstein and Legendre polynomials widely for solving system of linear and nonlinear second order BVP exploiting Galerkin weighted residual method.

\section{Some Special Polynomials}

In this section we give a short description on Bernstein [5] and Legendre [6] polynomials which are used in this paper.

\section{(a) Bernstein polynomials}

The general form of the Bernstein polynomials of nth degree over the interval $[a, b]$ is defined by [6]

$$
B_{i, n}(x)=\left(\begin{array}{c}
n \\
i
\end{array}\right) \frac{(x-a)^{i}(b-x)^{n-i}}{(b-a)^{n}}, a \leq x \leq b \quad i=0,1,2, \ldots \ldots \ldots \ldots, n
$$

Note that each of these $n+1$ polynomials having degree $n$ satisfies the following properties:

$$
\begin{array}{clc}
\text { i. } & B_{i, n}(x)=0, & \text { if } i<0 \text { or } i>n, \\
\text { ii. } & \sum_{i=0}^{n} B_{i, n}(x)=1 & \\
\text { iii. } & B_{i, n}(a)=B_{i, n}(b)=0, & 1 \leq i \leq n
\end{array}
$$

The first 11 Bernstein polynomials of degree ten over the interval $[0,1]$, are given below:

$$
\begin{aligned}
\text { i. } & B_{0,10}(x)=(1-x)^{10} \\
\text { ii. } & B_{1,10}(x)=10(1-x)^{9} x \\
\text { iii. } & B_{2,10}(x)=45(1-x)^{8} x^{2} \\
\text { iv. } & B_{3,10}(x)=120(1-x)^{7} x^{3} \\
\text { v. } & B_{4,10}(x)=210(1-x)^{6} x^{4} \\
\text { vi. } & B_{5,10}(x)=252(1-x)^{5} x^{5} \\
\text { vii. } & B_{6,10}(x)=210(1-x)^{4} x^{6} \\
\text { viii. } & B_{7,10}(x)=120(1-x)^{3} x^{7} \\
\text { ix. } & B_{8,10}(x)=45(1-x)^{2} x^{8} \\
\text { x. } & B_{9,10}(x)=10(1-x) x^{9} \\
\text { xi. } & B_{10,10}=x^{10}
\end{aligned}
$$

All these polynomial will satisfy the corresponding homogeneous form of the essential boundary conditions in the Galerkin method to solve a BVP.

\section{(b) Legendre Polynomials}

The general form of the Legendre polynomials [6] over the interval $[1,-1]$ is defined by

$$
p_{n}(x)=\sum_{r=0}^{N}(-1)^{r} \frac{(2 n-2 r) !}{2^{n} r !(n-r) !(n-2 r) !} x^{n-2 r}
$$


where $N=\frac{n}{2}$ for $n$ even and $N=\frac{n-1}{2}$ for $n$ odd.

The first ten Legendre polynomials are given below

$$
\begin{aligned}
& p_{1}(x)=x \\
& p_{2}(x)=\frac{1}{2}\left(3 x^{2}-1\right) \\
& p_{3}(x)=\frac{1}{2}\left(5 x^{3}-3 x\right) \\
& p_{4}(x)=\frac{1}{8}\left(35 x^{4}-30 x^{2}+3\right) \\
& p_{5}(x)=\frac{1}{8}\left(63 x^{5}-70 x^{3}+15 x\right) \\
& p_{6}(x)=\frac{1}{16}\left(231 x^{6}-315 x^{4}+105 x^{2}-5\right) \\
& p_{7}(x)=\frac{1}{16}\left(429 x^{7}-693 x^{5}+315 x^{3}-35 x\right) \\
& p_{8}(x)=\frac{1}{128}\left(6435 x^{8}-1201 x^{6}+6903 x^{4}-1260 x^{2}-35\right) \\
& p_{9}(x)=\frac{1}{128}\left(12155 x^{9}-25740 x^{7}+18018 x^{5}-4620 x^{3}+315 x\right) \\
& p_{10}(x)=\frac{1}{256}\left(46189 x^{10}-109395 x^{8}+90090 x^{6}-30030 x^{4}+3465 x^{2}-63\right)
\end{aligned}
$$

\section{System of Second Order Differential Equations}

General linear system of two second-order differential equations in two unknowns functions $u(x)$ and $v(x)$, is a system of the form [2]

$$
\left.\begin{array}{l}
a_{1}(x) u^{\prime \prime}+a_{2}(x) v^{\prime \prime}+a_{3}(x) u^{\prime}+a_{4}(x) v^{\prime}+a_{5}(x) u+a_{6}(x) v=f_{1}(x) \\
b_{1}(x) u^{\prime \prime}+b_{2}(x) v^{\prime \prime}+b_{3}(x) u^{\prime}+b_{4}(x) v^{\prime}+b_{5}(x) u+b_{6}(x) v=f_{2}(x)
\end{array}\right\}
$$

where $a_{i}(x), b_{i}(x), f_{1}(x), f_{2}(x)$ are given functions, and $a_{i}(x), b_{i}(x)$ are continuous, $i=1,2,3,4,5,6$

And general nonlinear system of two second-order differential equations in two unknowns functions $u(x)$ and $v(x)$, is a system of the form [4]

$$
\left.\begin{array}{c}
\left.a_{1}(x) u^{\prime \prime}+a_{2}(x) v^{\prime \prime}+a_{3}(x) u^{\prime}+a_{4}(x) v^{\prime}+a_{5}(x) u+a_{6}(x) v+N_{1}(u, v)=f_{1}(x)\right) \\
b_{1}(x) u^{\prime \prime}+b_{2}(x) v^{\prime \prime}+b_{3}(x) u^{\prime}+b_{4}(x) v^{\prime}+b_{5}(x) u+b_{6}(x) v+N_{2}(u, v)=f_{2}(x)
\end{array}\right\}
$$

where $a_{i}(x), b_{i}(x), f_{1}(x), f_{2}(x)$ are given functions, $N_{1}, N_{2}$ are nonlinear functions and $a_{i}(x), b_{i}(x)$ are continuous, $i=1,2,3,4,5,6$ 


\section{Formulation of Second Order BVP}

Let us consider the one dimensional system of second order differential equations [3]

$$
\left.\begin{array}{l}
-u^{\prime \prime}(x)+q(x) u(x)+r(x) v(x)=f(x) \\
-v^{\prime \prime}(x)+s(x) v(x)+t(x) u(x)=g(x)
\end{array}\right\}
$$

for the pair of functions $u(x)$ and $v(x)$ in $0<x<1$. Since each equation is of second order, two boundary conditions are required to specify each of the solution components $u(x)$ and $v(x)$ uniquely. For convenience, we assume homogeneous Dirichlet data at the ends as boundary conditions

$$
u(0)=u(1)=v(0)=v(1)=0
$$

The data include the prescribed functions $f, g, q, r, s$ and $t$, which are assumed to be bounded and sufficiently smooth to ensure subsequent variational integrals are well defined and the problem is “well posed”.

Let us consider two trial approximate solutions for the pair of functions $u(x)$ and $v(x)$ of system (1) given by

$$
\left.\begin{array}{l}
\tilde{u}(x)=\sum_{i=1}^{n} a_{i} p_{i}(x), n \geq 1 \\
\tilde{v}(x)=\sum_{i=1}^{n} b_{i} p_{i}(x), n \geq 1
\end{array}\right\}
$$

where $a_{i}$ and $b_{i}$ are parameter, $p_{i}(x)$ are co-ordinate functions (here Bernstein and Legendre polynomials) which satisfy boundary conditions (2).

Now apply Galerkin Method [1] in system (1) we get weighted residual system of equations

$$
\left.\begin{array}{l}
\int_{0}^{1}\left(-\tilde{u}^{\prime \prime}(x)+q(x) \tilde{u}(x)+r(x) \tilde{v}(x)\right) p_{i}(x) d x=\int_{0}^{1} f(x) p_{i}(x) d x \\
\int_{0}^{1}\left(-\tilde{u}^{\prime \prime}(x)+s(x) \tilde{u}(x)+t(x) \tilde{v}(x)\right) p_{i}(x) d x=\int_{0}^{1} f(x) p_{i}(x) d x
\end{array}\right\}
$$

Integrating by parts and setting $p_{i}(x)=0$ at the boundary $x=0$ and $x=1$, then we obtain system of weighted residual equations

$$
\left.\begin{array}{l}
\int_{0}^{1}\left(\tilde{u}^{\prime}(x) p_{i}^{\prime}(x)+q(x) \tilde{u}(x) p_{i}(x)+r(x) \tilde{v}(x) p_{i}(x)\right) d x=\int_{0}^{1} f(x) p_{i}(x) d x \\
\int_{0}^{1}\left(\tilde{v}^{\prime}(x) p_{i}^{\prime}(x)+s(x) \tilde{v}(x) p_{i}(x)+t(x) \tilde{u}(x) p_{i}(x)\right) d x=\int_{0}^{1} g(x) p_{i}(x) d x
\end{array}\right\}
$$

Now putting the representation (3) into (5) we get

$$
\begin{gathered}
\int_{0}^{1}\left(\sum_{j=1}^{n} a_{j} p_{j}{ }^{\prime}(x)\right. \\
\left.p_{i}^{\prime}(x)+q(x) \sum_{j=1}^{n} a_{j} p_{j}(x) p_{i}(x)+r(x) \sum_{j=1}^{n} b_{j} p_{j}(x) p_{i}(x)\right) d x \\
=\int_{0}^{1} f(x) p_{i}(x) d x
\end{gathered}
$$




$$
\begin{gathered}
\int_{0}^{1}\left(\sum_{j=1}^{n} b_{j} p_{j}{ }^{\prime}(x) p_{i}^{\prime}(x)+s(x) \sum_{j=1}^{n} b_{j} p_{j}(x) p_{i}(x)+t(x) \sum_{j=1}^{n} a_{j} p_{j}(x) p_{i}(x)\right) d x \\
=\int_{0}^{1} g(x) p_{i}(x) d x
\end{gathered}
$$

We can write above equation as

$$
\begin{gathered}
\sum_{j=1}^{n}\left(\int_{0}^{1}\left[\left(p_{j}^{\prime}(x) p_{i}^{\prime}(x)\right) a_{j}+\left(q(x) p_{j}(x) p_{i}(x)\right) a_{j}+\left(r(x) p_{j}(x) p_{i}(x)\right) b_{j}\right] d x\right) \\
=\int_{0}^{1} f(x) p_{i}(x) d x \\
\sum_{j=1}^{n}\left(\int_{0}^{1}\left[\left(p_{j}^{\prime}(x) p_{i}^{\prime}(x)\right) b_{j}+\left(s(x) p_{j}(x) p_{i}(x)\right) b_{j}+\left(t(x) p_{j}(x) p_{i}(x)\right) a_{j}\right] d x\right) \\
=\int_{0}^{1} g(x) p_{i}(x) d x \\
i=1,2,3, \ldots, n
\end{gathered}
$$

Equivalently,

$$
\left.\begin{array}{l}
\sum_{j=1}^{n}\left(A_{j, i} a_{j}+B_{j, i} a_{j}\right)=F_{i} \\
\sum_{j=1}^{n}\left(C_{j, i} b_{j}+D_{j, i} a_{j}\right)=G_{i} \\
i=1,2,3, \ldots, n
\end{array}\right\}
$$

where,

$$
\begin{aligned}
A_{j, i} & =\int_{0}^{1}\left[\left(p_{j}^{\prime}(x) p_{i}^{\prime}(x)\right)+\left(q(x) p_{j}(x) p_{i}(x)\right)\right] d x \\
B_{j, i} & =\int_{0}^{1}\left(r(x) p_{j}(x) p_{i}(x)\right) d x \\
F_{i} & =\int_{0}^{1} f(x) p_{i}(x) d x \\
C_{j, i} & =\int_{0}^{1}\left[\left(p_{j}^{\prime}(x) p_{i}^{\prime}(x)\right)+\left(s(x) p_{j}(x) p_{i}(x)\right)\right] d x \\
D_{j, i} & =\int_{0}^{1}\left(t(x) p_{j}(x) p_{i}(x)\right) d x \\
G_{i} & =\int_{0}^{1} g(x) p_{i}(x) d x \\
i, j & =1,2,3, \ldots, n
\end{aligned}
$$


For $i=1,2, \ldots, n$ we get system of linear equations, which involve parameter $a_{i}$ and $b_{i}$ which can be obtained by solving system (6). System (6) can be assembled by element matrix contribution [3]. Since there is no direct method to solve nonlinear BVPs, so we describe the proposed method for nonlinear BVPs through numerical examples in the next section.

\section{Numerical Examples}

In this study, we use three BVPs; two linear and one nonlinear, which are available in the existing literature [4], the Dirichlet boundary conditions are considered to verify the effectiveness of the derived formulations. For each case we find the approximate solutions using different number of parameters with Bernstein and Legendre polynomials, and we compare these solutions with the exact solutions, and graphically which are shown in the same diagram.

\section{Example 1}

Consider the following system of equations [4]

$$
\left.\begin{array}{c}
u^{\prime \prime}(x)+x u(x)+x v(x)=2 \\
v^{\prime \prime}(x)+2 x v(x)+2 x u(x)=-2
\end{array}\right\}
$$

subject to the boundary conditions

$u(0)=u(1)=0, \quad v(0)=v(1)=0$

where $0<x<1$. The exact solution of (7) are $u(x)=x^{2}-x$ and $v(x)=x-x^{2}$.

\section{Solutions using Bernstein polynomials:}

We use Bernstein polynomials as trial solution to solve the system (7). Consider trial approximate solutions be

$$
\left.\begin{array}{l}
\tilde{u}(x)=\sum_{i=1}^{n} a_{i} B_{i, 10}(x) \\
\tilde{v}(x)=\sum_{i=1}^{n} b_{i} B_{i, 10}(x)
\end{array}\right\}
$$

where $a_{i}$ and $b_{i}$ are parameter and $B_{i, 10}(x)$ are co-ordinate functions of Bernstein polynomials which satisfy conditions (8).

Using the method illustrated in section 4, finally we get,

$$
\begin{aligned}
& \sum_{j=1}^{n}\left(\int_{0}^{1}\left[\left(-B_{j, 10}^{\prime}(x) B_{i, 10}^{\prime}(x)\right) a_{j}+\left(x B_{j, 10}(x) B_{i, 10}(x)\right) a_{j}+\left(x B_{j, 10}(x) B_{i, 10}(x)\right) b_{j}\right] d x\right) \\
& =\int_{0}^{1} f(x) B_{i, 10}(x) d x
\end{aligned}
$$




$$
\begin{aligned}
& \sum_{j=1}^{n}\left(\int_{0}^{1}\left[\left(-B_{j, 10}^{\prime}(x) B_{i, 10}^{\prime}(x)\right) b_{j}+\left(2 x B_{j, 10}(x) B_{i, 10}(x)\right) b_{j}+\left(2 x B_{j, 10}(x) B_{i, 10}(x)\right) a_{j}\right] d x\right) \\
& =\int_{0}^{1} g(x) B_{i, 10}(x) d x \\
& i=1,2, \ldots, n
\end{aligned}
$$

The above equations are equivalent to the matrix form

$$
\begin{aligned}
& \sum_{j=1}^{n}\left(A_{j, i} a_{j}+H_{j, i} a_{j}\right)=F_{i} \\
& \sum_{j=1}^{n}\left(C_{j, i} b_{j}+D_{j, i} a_{j}\right)=G_{i}
\end{aligned}
$$

where,

$$
\begin{aligned}
A_{j, i} & =\int_{0}^{1}\left[\left(-B_{j, 10}^{\prime}(x) B_{i, 10}^{\prime}(x)\right)+\left(x B_{j, 10}(x) B_{i, 10}(x)\right)\right] d x \\
H_{j, i} & =\int_{0}^{1}\left(x B_{j, 10}(x) B_{i, 10}(x)\right) d x \\
F_{i} & =\int_{0}^{1} 2(x) B_{i, 10}(x) d x \\
C_{j, i} & =\int_{0}^{1}\left[\left(-B_{j, 10}^{\prime}(x) B_{i, 10}^{\prime}(x)\right)+\left(2 x B_{j, 10}(x) B_{i, 10}(x)\right)\right] d x \\
D_{j, i} & =\int_{0}^{1}\left(2 x B_{j, 10}(x) B_{i, 10}(x)\right) d x \\
G_{i} & =\int_{0}^{1}-2 B_{i, 10}(x) d x \quad i, j=1,2, \ldots, n
\end{aligned}
$$

Similarly, we can derive the equation (10) using Legendre polynomials.

Table 1: Results of $\breve{u}(x)$ of equation (7) in Example 1

\begin{tabular}{|c|c|c|c|c|c|c|}
\hline \multirow{2}{*}{$\mathbf{x}$} & Exact value & $\begin{array}{c}\text { Approximate } \\
\text { Solution } \mathbf{u}(\mathbf{x})\end{array}$ & Absolute error & $\begin{array}{c}\text { Approximate } \\
\text { Solution } \mathbf{u}(\mathbf{x})\end{array}$ & $\begin{array}{c}\text { Absolute error } \\
\text { Legendre polynomial } \mathrm{n}=2\end{array}$ & $\begin{array}{c}\text { Absolute error } \\
{[4]}\end{array}$ \\
\cline { 3 - 8 } & & \multicolumn{2}{|c|}{ Bernstein polynomial $\mathrm{n}=9$} & \\
\hline $\mathbf{0 . 0}$ & 0.0000000000 & 0.0000000000 & 0.0000000000 & 0.000000000 & 0.0000000000 & $\mathbf{0 . 0 0 0 0 0 0 0 0 0 0}$ \\
$\mathbf{0 . 1}$ & -0.0900000000 & -0.0900000000 & $6.9388939 \times 10^{-18}$ & -0.09000000 & $2.8008740130 \times 10^{-11}$ & $\mathbf{0 . 0 0 0 0 0 0 0 0 0 0}$ \\
$\mathbf{0 . 2}$ & -0.1600000000 & -0.1600000000 & $2.7755575 \times 10^{-17}$ & -0.16000000 & $3.6821549280 \times 10^{-11}$ & $\mathbf{0 . 0 0 0 0 0 0 0 0 0 0}$ \\
$\mathbf{0 . 3}$ & -0.2100000000 & -0.2100000000 & 0.0000000000 & -0.21000000 & $6.2091685960 \times 10^{-11}$ & $\mathbf{0 . 0 0 0 0 0 0 0 0 0 0}$ \\
$\mathbf{0 . 4}$ & -0.2400000000 & -0.2400000000 & 0.0000000000 & -0.24000000 & $1.0877099220 \times 10^{-11}$ & $\mathbf{0 . 0 0 0 0 0 0 0 0 0 0}$ \\
$\mathbf{0 . 5}$ & -0.2500000000 & -0.2500000000 & 0.0000000000 & -0.25000000 & $1.3281251100 \times 10^{-11}$ & $\mathbf{0 . 0 0 0 0 0 0 0 0 0 0}$ \\
$\mathbf{0 . 6}$ & -0.2400000000 & -0.2400000000 & 0.0000000000 & -0.24000000 & $1.0877088120 \times 10^{-11}$ & $\mathbf{0 . 0 0 0 0 0 0 0 0 0 0}$ \\
$\mathbf{0 . 7}$ & -0.2100000000 & -0.2100000000 & 0.0000000000 & -0.16000000 & $6.2091748410 \times 10^{-11}$ & $\mathbf{0 . 0 0 0 0 0 0 0 0 0 0}$ \\
$\mathbf{0 . 8}$ & -0.1600000000 & -0.1600000000 & 0.0000000000 & -0.16000000 & $3.6821518060 \times 10^{-11}$ & $\mathbf{0 . 0 0 0 0 0 0 0 0 0 0}$ \\
$\mathbf{0 . 9}$ & -0.0900000000 & -0.0900000000 & 0.0000000000 & -0.09000000 & $2.8008692590 \times 10^{-11}$ & $\mathbf{0 . 0 0 0 0 0 0 0 0 0 0}$ \\
$\mathbf{1 . 0}$ & 0.0000000000 & 0.0000000000 & 0.0000000000 & 0.000000000 & 0.0000000000 & $\mathbf{0 . 0 0 0 0 0 0 0 0 0 0}$ \\
\hline
\end{tabular}




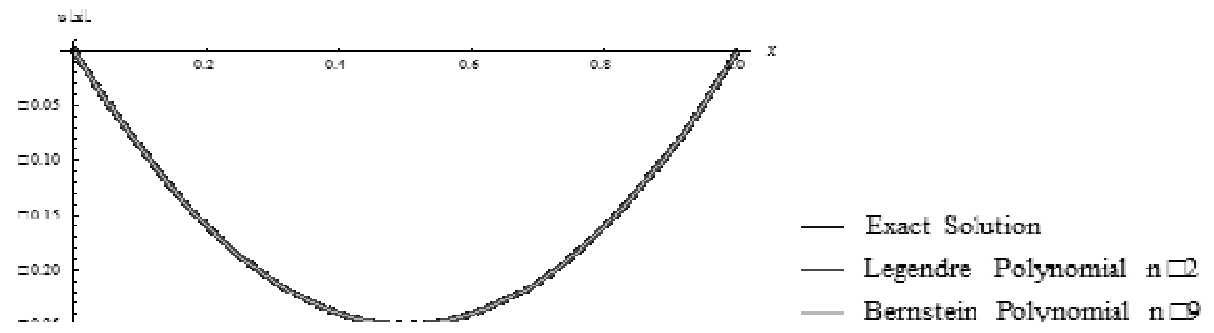

Figure 1: Graphical representation of exact and approximate solutions of $u(x)$ of equation (7)

Table 2: Results of $v\{(x)$ of equation (7) in example 1

\begin{tabular}{|c|c|c|c|c|c|c|}
\hline \multirow[t]{2}{*}{$x$} & \multirow[t]{2}{*}{ Exact value } & $\begin{array}{l}\text { Approximate } \\
\text { Solution } v(x)\end{array}$ & Absolute error & $\begin{array}{l}\text { Approximate } \\
\text { Solution } v(x)\end{array}$ & Absolute error & \multirow[t]{2}{*}{$\begin{array}{c}\text { Absolute error } \\
\text { [4] }\end{array}$} \\
\hline & & \multicolumn{2}{|c|}{ Legendre polynomial $n=2$} & \multicolumn{2}{|c|}{ Bernstein polynomial $n=9$} & \\
\hline 0.0 & 0.0000000000 & 0.0000000000 & 0.0000000000 & 0.0000000000 & 0.0000000000 & 0.0000000000 \\
\hline 0.1 & 0.0900000000 & 0.0900000000 & $6.9388939 \times 10^{-18}$ & 0.0900000000 & $2.8008740130 \times 10^{-11}$ & 0.0000000000 \\
\hline 0.2 & 0.1600000000 & 0.1600000000 & $2.7755575 \times 10^{-17}$ & 0.1600000000 & $3.6821549280 \times 10^{-11}$ & 0.0000000000 \\
\hline 0.3 & 0.2100000000 & 0.2100000000 & 0.0000000000 & 0.2100000000 & $6.2091685960 \times 10^{-11}$ & 0.0000000000 \\
\hline 0.4 & 0.2400000000 & 0.2400000000 & 0.0000000000 & 0.2400000000 & $1.0877099220 \times 10^{-11}$ & 0.0000000000 \\
\hline 0.5 & 0.2500000000 & 0.2500000000 & 0.0000000000 & 0.2500000000 & $1.3281251100 \times 10^{-11}$ & 0.0000000000 \\
\hline 0.6 & 0.2400000000 & 0.2400000000 & 0.0000000000 & 0.2400000000 & $1.0877088120 \times 10^{-11}$ & 0.0000000000 \\
\hline 0.7 & 0.2100000000 & 0.2100000000 & 0.0000000000 & 0.1600000000 & $6.2091748410 \times 10^{-11}$ & 0.0000000000 \\
\hline 0.8 & 0.1600000000 & 0.1600000000 & 0.0000000000 & 0.1600000000 & $3.6821518060 \times 10^{-11}$ & 0.0000000000 \\
\hline 0.9 & 0.0900000000 & 0.0900000000 & 0.0000000000 & 0.0900000000 & $2.8008692590 \times 10^{-11}$ & 0.0000000000 \\
\hline 1.0 & 0.0000000000 & 0.0000000000 & 0.0000000000 & 0.0000000000 & 0.0000000000 & 0.0000000000 \\
\hline
\end{tabular}

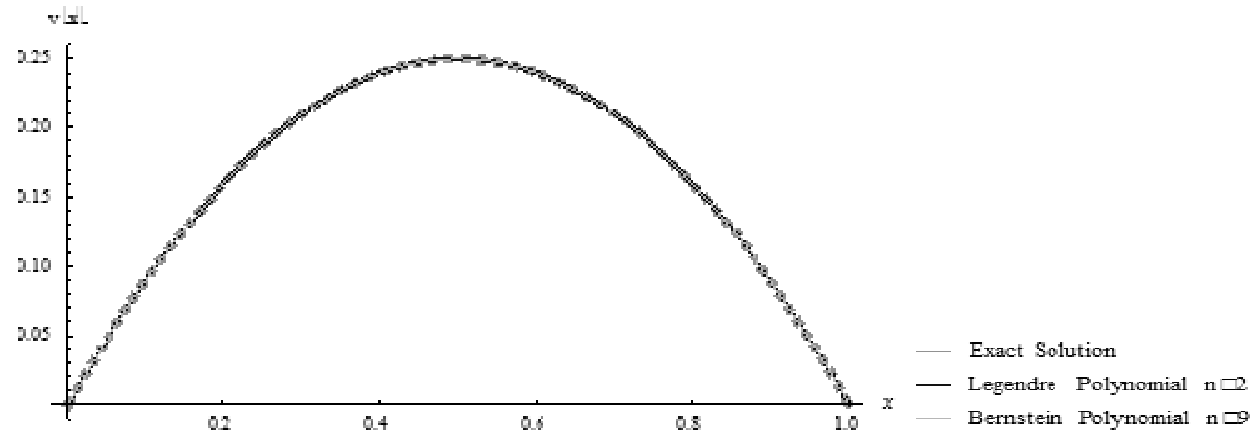

Figure 2: Graphical representation of exact and approximate solutions of $v(x)$ of equation (7)

\section{Example 2:}

Consider the following system of equations [4] 
$\left.u^{\prime \prime}(x)+(2 x-1) u^{\prime}(x)+\cos (\pi x) v^{\prime}(x)=-\pi^{2} \sin (\pi x)+(2 x-1) \pi \cos (\pi x)\right\}$

$v^{\prime \prime}(x)+x u(x)=2+x \sin (\pi x)$

subject to the boundary conditions

$u(0)=u(1)=0, v(0)=v(1)=0$

where $0<x<1$. The exact solutions of (11) are $u(x)=\sin (\pi x)$ and $v(x)=x^{2}-x$.

Using the same procedure of example 1 we get the following Table 3 and graphs.

Table 3: Results of $\breve{u}(x)$ of equation (11)

\begin{tabular}{|c|c|c|c|c|c|c|c|}
\hline \multirow{2}{*}{$x$} & \multirow{2}{*}{ Exact value } & $\begin{array}{c}\text { Approximate } \\
\text { Solution } v(x)\end{array}$ & Absolute error & $\begin{array}{c}\text { Approximate } \\
\text { Solution } v(x)\end{array}$ & Absolute error & $\begin{array}{c}\text { Absolute error } \\
{[4]}\end{array}$ \\
\cline { 3 - 7 } & & \multicolumn{2}{|c|}{ Legendre polynomial n=3 } & \multicolumn{2}{|c|}{ Bernstein polynomial $\mathrm{n}=8$} & \\
\hline 0.0 & 0.0000000000 & 0.0000000000 & 0.0000000000 & 0.0000000000 & 0.0000000000 & 0.0000000000 \\
\hline 0.1 & 0.3090169944 & 0.3087639360 & $2.53058374 \times 10^{-4}$ & 0.3215460957 & $1.252910136 \times 10^{-2}$ & $3.00000000 \times 10^{-4}$ \\
\hline 0.2 & 0.5877852523 & 0.8095597440 & $5.42749625 \times 10^{-4}$ & 0.8230835958 & $1.406660147 \times 10^{-2}$ & $2.50000 \times 00010^{-3}$ \\
\hline 0.3 & 0.8090169944 & 0.8095597440 & $5.42749625 \times 10^{-4}$ & .08230835958 & $1.406660147 \times 10^{-2}$ & $7.80000000 \times 10^{-3}$ \\
\hline 0.4 & 0.9510565163 & 0.9506716800 & $3.84836395 \times 10^{-4}$ & 0.9507983300 & $2.5186306 \times 10^{-4}$ & $1.66000000 \times 10^{-2}$ \\
\hline 0.5 & 1.0000000000 & 0.9991240000 & $8.76000000 \times 10^{-4}$ & 0.9691424805 & $3.085751953 \times 10^{-2}$ & $2.77000000 \times 10^{-2}$ \\
\hline 0.6 & 0.9510565163 & 0.950673160 & $3.83300295 \times 10^{-4}$ & 0.9539908751 & $2.93435882 \times 10^{-3}$ & $3.87000000 \times 10^{-2}$ \\
\hline 0.7 & 0.8090169944 & 0.8095624320 & $5.45437625 \times 10^{-4}$ & 0.8134388915 & $4.42189715 \times 10^{-3}$ & $4.59000000 \times 10^{-2}$ \\
\hline 0.8 & 0.5877852523 & 0.5885213440 & $7.36091707 \times 10^{-4}$ & 0.5536130521 & $3.417220016 \times 10^{-2}$ & $4.49000000 \times 10^{-2}$ \\
\hline 0.9 & 0.3090169944 & 0.3087662400 & $2.50754374 \times 10^{-4}$ & 0.3147098746 & $5.692880216 \times 10^{-3}$ & $3.09000000 \times 10^{-2}$ \\
\hline 1.0 & 0.0000000000 & 0.0000000000 & 0.0000000000 & 0.0000000000 & 0.0000000000 & 0.0000000000 \\
\hline
\end{tabular}

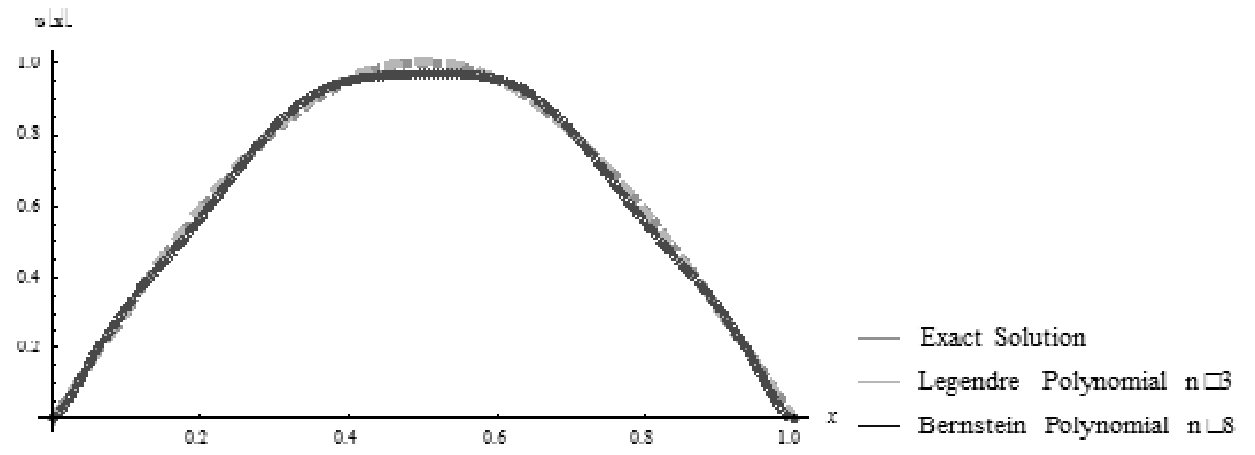

Figure 3: Graphical representation of exact and approximate solutions of $u(x)$ of equation (11) 
Table 4: Results of $v\{(x)$ of equation (11)

\begin{tabular}{|c|c|c|c|c|c|c|}
\hline \multirow[t]{2}{*}{$x$} & \multirow[t]{2}{*}{ Exact value } & $\begin{array}{l}\text { Approximate } \\
\text { Solution } v(x)\end{array}$ & Absolute error & $\begin{array}{l}\text { Approximate } \\
\text { Solution } v(x)\end{array}$ & Absolute error & \multirow[t]{2}{*}{$\begin{array}{c}\text { Absolute error } \\
{[4]}\end{array}$} \\
\hline & & \multicolumn{2}{|c|}{ Legendre polynomial $n=3$} & \multicolumn{2}{|c|}{ Bernstein polynomial $n=4$} & \\
\hline 0.0 & 0.0000000000 & 0.0000000000 & 0.0000000000 & 0.0000000000 & 0.0000000000 & 0.0000000000 \\
\hline 0.1 & 0.3090169944 & 0.3087639360 & $2.53058374 \times 10^{-4}$ & 0.3215460957 & $1.252910136 \times 10^{-2}$ & $3.00000000 \times 10^{-4}$ \\
\hline 0.2 & 0.5877852523 & 0.8095597440 & $5.42749625 \times 10^{-4}$ & 0.8230835958 & $1.406660147 \times 10^{-2}$ & $2.50000 \times 00010^{-3}$ \\
\hline 0.3 & 0.8090169944 & 0.8095597440 & $5.42749625 \times 10^{-4}$ & 0.8230835958 & $1.406660147 \times 10^{-2}$ & $7.80000000 \times 10^{-3}$ \\
\hline 0.4 & 0.9510565163 & 0.9506716800 & $3.84836395 \times 10^{-4}$ & 0.9507983300 & $2.5186306 \times 10^{-4}$ & $1.66000000 \times 10^{-2}$ \\
\hline 0.5 & 1.0000000000 & 0.950673160 & $8.76000000 \times 10^{-4}$ & 0.9691424805 & $3.085751953 \times 10^{-2}$ & $2.77000000 \times 10^{-2}$ \\
\hline 0.6 & 0.9510565163 & 0.950673160 & $3.83300295 \times 10^{-4}$ & 0.9539908751 & $2.93435882 \times 10^{-3}$ & $3.87000000 \times 10^{-2}$ \\
\hline 0.7 & 0.8090169944 & 0.8095624320 & $5.45437625 \times 10^{-4}$ & 0.8134388915 & $4.42189715 \times 10^{-3}$ & $4.59000000 \times 10^{-2}$ \\
\hline 0.8 & 0.5877852523 & 0.5885213440 & $7.36091707 \times 10^{-4}$ & 0.5536130521 & $3.417220016 \times 10^{-2}$ & $4.49000000 \times 10^{-2}$ \\
\hline 0.9 & 0.3090169944 & 0.3087662400 & $2.50754374 \times 10^{-4}$ & 0.3147098746 & $5.692880216 \times 10^{-3}$ & $3.09000000 \times 10^{-2}$ \\
\hline 1.0 & 0.0000000000 & 0.0000000000 & 0.0000000000 & 0.0000000000 & 0.0000000000 & 0.0000000000 \\
\hline
\end{tabular}
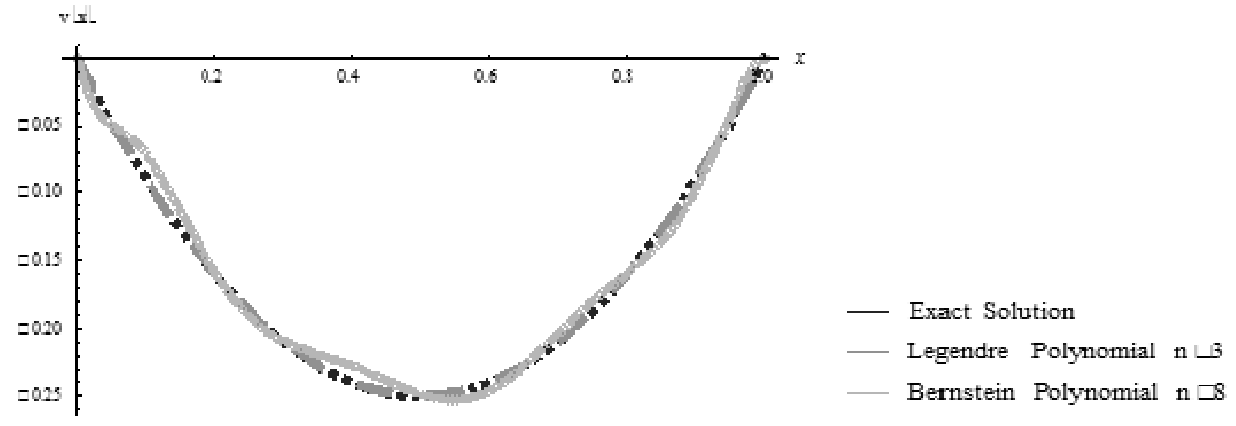

Figure 4: Graphical representation of exact and approximate solutions of $v(x)$ of equation (11)

\section{Example 3 :}

Consider the following equations [4]

$u^{\prime \prime}(x)+x u^{\prime}(x)+\cos (\pi x) v^{\prime}(x)=\sin (x)+\left(x^{2}-x+2\right) \cos (x)+(1-2 x) \cos (\pi x)$

$v^{\prime \prime}(x)+x u^{\prime}(x)+u^{2}(x)=-2+x \sin (x)+x(x-1)^{2} \sin ^{2}(x)+\left(x^{2}-x\right) \cos (x) . \quad$

subject to the boundary condition

$u(0)=u(1)=0, \quad v(0)=v(1)=0$

where $0<x<1$. The exact solutions of (13) are $u(x)=(x-1) \sin (x)$ and $v(x)=x-x^{2}$.

We use Legendre polynomials as trial approximate solution to solve the system (13). Consider trial solution be of the form 


$$
\left.\begin{array}{l}
\tilde{u}(x)=\sum_{i=1}^{n} b_{i} L_{i}(x) \\
\tilde{v}(x)=\sum_{i=1}^{n} b_{i} L_{i}(x)
\end{array}\right\}
$$

where $a_{i}$ and $b_{i}$ are parameter $L_{i}(x)$ are co-ordinate functions of Legendre polynomials which satisfy conditions (14).

Using the method illustrated in section 4, finally we get

$$
\begin{aligned}
& \sum_{i=1}^{n}\left(\int_{0}^{1}\left[\left(-L_{j}^{\prime}(x) L_{i}^{\prime}(x)\right) a_{i}+\left(\left(x L_{j}(x) L_{i}^{\prime}(x)\right) a_{i}+\left(\cos (\pi x) L_{i}^{\prime}(x) L_{j}(x)\right) b_{i}\right] d x\right)\right. \\
& =\int_{0}^{1} f_{1}(x) L_{j}(x) d x \\
& \sum_{i=1}^{n}\left(\int_{0}^{1}\left(\left(-L_{j}^{\prime}(x) L_{i}^{\prime}(x)\right) b_{i}+\left(\left(x L_{j}(x) L_{i}^{\prime}(x)\right)+\sum_{k=1}^{2} a_{k} L^{2}{ }_{k}(x) L_{j}(x)\right) a_{i}\right) d x\right) \\
& =\int_{0}^{1} f_{2}(x) L_{j}(x) d x \quad i, j=1,2, \ldots \ldots \ldots, n
\end{aligned}
$$

The above equations are equivalent to the matrix form

$$
\begin{aligned}
& \sum_{i=1}^{n}\left(A_{i, j} a_{i}+B_{i, j} a_{i}\right)=F_{j} \\
& \sum_{i=1}^{n}\left(C_{i, j} b_{i}+\left(D_{i, j}+E_{i, j}\right) a_{i}\right)=G_{j}
\end{aligned}
$$

where,

$$
\begin{aligned}
& A_{i, j}=\int_{0}^{1}\left(\left(-L_{j}^{\prime}(x) L_{i}^{\prime}(x)\right)+\left(\left(x L_{i}^{\prime}(x) L_{j}(x)\right)\right) d x\right. \\
& B_{i, j}=\int_{0}^{1}\left(\cos (\pi x) L_{i}^{\prime}(x) L_{j}(x)\right) d x \\
& F_{j}=\int_{0}^{1}\left(\sin (x)+\left(x^{2}-x+2\right) \cos (x)+(1-2 x) \cos (\pi x)\right) L_{j}(x) d x \\
& C_{i, j}=\int_{0}^{1}\left(-L_{j}^{\prime}(x) L_{i}^{\prime}(x)\right) d x \\
& D_{i, j}=\int_{0}^{1}\left(x L(x) L_{i}^{\prime}(x)\right) d x \\
& E_{i, j}=\int_{0}^{1}\left(\sum_{k=1}^{n} a_{k} L_{k}^{2}(x) L_{j}(x)\right) d x
\end{aligned}
$$




$$
\begin{aligned}
& G_{i}=\int_{0}^{1}\left(-2+x \sin (x)+x(x-1)^{2} \sin ^{2}(x)+\left(x^{2}-x\right) \cos (x)\right) L_{j}(x) d x \\
& i, j=1,2, \ldots \ldots \ldots, n .
\end{aligned}
$$

The initial values of these coefficients $a_{i}$ are obtained by applying Galerkin method to the BVP neglecting the nonlinear term in $17(b)$. That is, to find initial coefficients we will solve the system

$$
\left.\begin{array}{c}
\sum_{i=1}^{n}\left\{A_{i, j} a_{i}+B_{i, j} a_{i}\right\}=F_{j} \\
\sum_{i=1}^{n}\left\{C_{i, j} b_{i}+D_{i, j} a_{i}\right\}=G_{j}
\end{array}\right\}
$$

whose matrices are constructed from

$$
\begin{aligned}
A_{i, j} & =\int_{0}^{1}\left(\left(-L_{j}^{\prime}(x) L_{i}^{\prime}(x)\right)+\left(\left(x L_{i}^{\prime}(x) L_{j}(x)\right)\right) d x\right. \\
B_{i, j} & =\int_{0}^{1}\left(\cos (\pi x) L_{i}^{\prime}(x) L_{j}(x)\right) d x \\
F_{j} & =\int_{0}^{1}\left(\sin (x)+\left(x^{2}-x+2\right) \cos (x)+(1-2 x) \cos (\pi x)\right) L_{j}(x) d x
\end{aligned}
$$

Table 5: Results of $\breve{u}(x)$ of equation (13)

\begin{tabular}{|c|c|c|c|c|c|c|}
\hline$x$ & \multirow{2}{*}{ Exact value } & $\begin{array}{c}\text { Approximate } \\
\text { Solution } v(x)\end{array}$ & Absolute error & $\begin{array}{c}\text { Approximate } \\
\text { Solution } v(x)\end{array}$ & Absolute error & $\begin{array}{c}\text { Absolute error } \\
{[4]}\end{array}$ \\
\cline { 3 - 6 } & & \multicolumn{2}{|c|}{ Legendre polynomial $\mathrm{n}=2$} & \multicolumn{2}{|c|}{ Bernstein polynomial $\mathrm{n}=9$} & \\
\hline 0.0 & 0.0000000000 & 0.0000000000 & 0.0000000000 & 0.0000000000 & 0.0000000000 & 0.0000000000 \\
\hline 0.1 & -0.0898500750 & 0.0000000000 & $1.421902218 \times 10^{-3}$ & -0.0898555699 & $5.4949000000 \times 10^{-6}$ & $3.00000 \times 10^{-4}$ \\
\hline 0.2 & -0.1589354646 & -0.0912719772 & $7.683689640 \times 10^{-4}$ & -0.1589352157 & $2.489000000 \times 10^{-7}$ & $2.50000 \times 10^{-3}$ \\
\hline 0.3 & -0.206641447 & -0.1597038336 & $6.095282628 \times 10^{-4}$ & -0.2068641998 & $5.51000000 \times 10^{-8}$ & $7.80000 \times 10^{-3}$ \\
\hline 0.4 & -0.2336510054 & -02062546164 & $1.767632585 \times 10^{-3}$ & -0.2336500057 & $9.9970000 \times 10^{-7}$ & $1.66000 \times 10^{-2}$ \\
\hline 0.5 & -0.2397127693 & -0.2318833728 & $2.163619305 \times 10^{-3}$ & -0.2397123941 & $3.75200000 \times 10^{-7}$ & $2.77000 \times 10^{-2}$ \\
\hline 0.6 & -0.2258569894 & -0.2375491500 & $1.645994158 \times 10^{-3}$ & -0.2258523662 & $4.62320000 \times 10^{-6}$ & $3.87000 \times 10^{-2}$ \\
\hline 0.7 & -0.1932653062 & -0.2242109952 & $4.373505713 \times 10^{-4}$ & -0.1932605023 & $4.80390000 \times 10^{-6}$ & $4.59000 \times 10^{-2}$ \\
\hline 0.8 & -1434712182 & -0.1928279556 & $8.878602201 \times 10^{-4}$ & -0.1434700211 & $1.19710000 \times 10^{-6}$ & $4.49000 \times 10^{-2}$ \\
\hline 0.9 & -0.0783326910 & -0.1443590784 & $1.430719837 \times 10^{-3}$ & -0.0783311456 & $1.54540000 \times 10^{-6}$ & $3.09000 \times 10^{-2}$ \\
\hline 1.0 & 0.0000000000 & 0.0000000000 & 0.0000000000 & 0.0000000000 & 0.0000000000 & 0.0000000000 \\
\hline
\end{tabular}

$$
C_{i, j}=\int_{0}^{1}\left(-p_{j}^{\prime}(x) L_{i}^{\prime}(x)\right) d x
$$




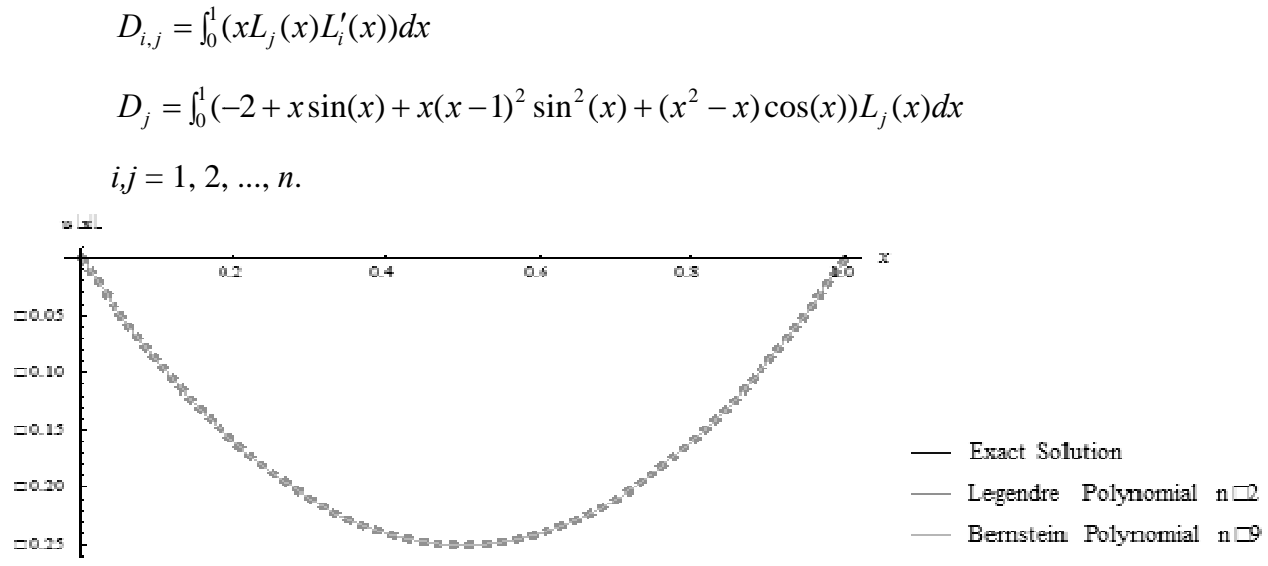

Figure 5: Graphical representation of exact and approximate solutions of $u(x)$ of equation (13)

Table 6: Results of $v \diamond(x)$ of equation (13) using

\begin{tabular}{|c|c|c|c|c|c|c|}
\hline \multirow[t]{2}{*}{$x$} & \multirow[t]{2}{*}{ Exact value } & $\begin{array}{l}\text { Approximate } \\
\text { Solution } v(x)\end{array}$ & Absolute error & $\begin{array}{l}\text { Approximate } \\
\text { Solution } v(x)\end{array}$ & Absolute error & \multirow[t]{2}{*}{$\begin{array}{c}\text { Absolute error } \\
\text { [4] }\end{array}$} \\
\hline & & \multicolumn{2}{|c|}{ Legendre polynomial $n=2$} & \multicolumn{2}{|c|}{ Bernstein polynomial n=9 } & \\
\hline 0.0 & 0.0000000000 & 0.0000000000 & 0.0000000000 & 0.0000000000 & 0.0000000000 & 0.0000000000 \\
\hline 0.1 & 0.0900000000 & 0.0891388543 & $8.61145740 \times 10^{-4}$ & 0.0899952168 & $4.78320000 \times 10^{-6}$ & 0.0000000000 \\
\hline 0.2 & 0.1600000000 & 1.1593721549 & $6.27845120 \times 10^{-4}$ & 0.1600002894 & $2.89400000 \times 10^{-7}$ & 0.0000000000 \\
\hline 0.3 & 0.2100000000 & 0.2103612466 & $3.61246620 \times 10^{-4}$ & 0.21009999877 & $9.99877000 \times 10^{-5}$ & 0.0000000000 \\
\hline 0.4 & 0.2400000000 & 0.2417674742 & $1.76747424 \times 10^{-2}$ & 0.239999999998 & $2.0000000 \times 10^{-10}$ & 0.0000000000 \\
\hline 0.5 & 0.2500000000 & 0.2532521825 & $3.25218250 \times 10^{-3}$ & 0.2511119953 & $9.95300000 \times 10^{-7}$ & 0.0000000000 \\
\hline 0.6 & 0.2400000000 & 0.2444767162 & $4.47671616 \times 10^{-2}$ & 0.2400001511 & $1.51100000 \times 10^{-7}$ & 0.0000000000 \\
\hline 0.7 & 0.2100000000 & 0.2151024200 & $5.10241998 \times 10^{-2}$ & 0.2099999899 & $1.01000000 \times 10^{-8}$ & 0.0000000000 \\
\hline 0.8 & 0.1600000000 & 0.1647906387 & $4.79063872 \times 10^{-3}$ & 0.1599879652 & $1.20348000 \times 10^{-5}$ & 0.0000000000 \\
\hline 0.9 & 0.0900000000 & 0.0932027171 & $3.20271714 \times 10^{-3}$ & 0.0900501123 & $5.01123000 \times 10^{-5}$ & 0.0000000000 \\
\hline 1.0 & 0.0000000000 & 0.0000000000 & 0.0000000000 & 0.0000000000 & 0.0000000000 & 0.0000000000 \\
\hline
\end{tabular}

Once the initial values of the coefficients $a_{i}$ are obtained from the system $17(c)$, they are substituted into equation $17(b)$ to obtain new estimates for the values of $a_{i}$. This iteration process continues until the converged values of the unknown parameters are obtained. Substituting the final values of the parameters into (14), we obtain an approximate solution of the BVP (13).

\section{Conclusions}

We have derived, in details, the formulation of system of second order BVPs by Galerkin weighted residual method. This method enables us to approximate the solutions at every point of the domain of the problem. In this method, we have used Legendre and Bernstein polynomials as the trial functions in the approximation. The concentration has given not only on the performance of the results but also on the formulations. 


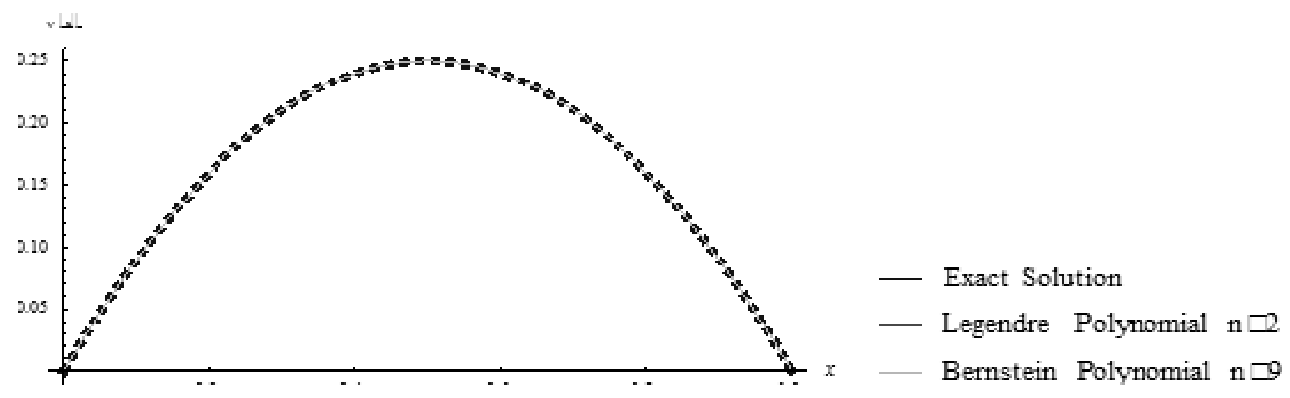

Figure 6: Graphical representation of exact and approximate solutions of $v(x)$ of equation (13)

We may notice that the formulations of this study are easy to understand and may be implemented to solve for BVP. Some linear and nonlinear examples are tested to verify the effectiveness of the desired formulations whose analytical solutions are not available. The computed solutions are compared with the exact solutions and we have found a good agreement. This method may be applied for higher order BVPs to get the desired accuracy.

\section{REFERENCES}

[1] M. Wazwaz, A new method for solving singular initial problems in the second order ordinary differential equations, Appl. Math. Comput. 128 (2002) 45-57.

[2] J.I. Ramos, Linearization techniques for singular initial value problems of ordinary differential equations, Appl. Math. Comput. 161 (2005) 525-542.

[3] Eric B. Becker, Graham F, Carey and J. Tinsley Oden, FINITE ELEMENTS An Introduction, Volume I, Prentice-Hall, Inc (1981).

[4] Jungfeng Lu, Variational Iteration Method for Solving a nonlinear System of Second-order Boundary value problems, Computer Mathematics with Applications, 54(2007), 1133-1138.

[5] M.I. Bhatti and P. Bracken, Solutions of Differential Equations in a Bernstein Polynomials Basis, Jn. Comput. Appl. Math. 205 (2007), 272-280.

[6] Md. Shafiqul Islam, Md. Bellal Hossain, On the use of piecewise standard polynomials in the numerical solutions of fourth order boundary value problems, GANIT Jn. Bangladesh Math. Soc. Vol. 33(2013) 53-64.

[7] Gear, C.W. Numerical initial value problems in ordinary differential equations, Prentice-Hall, Englewood Cliffs, NJ (1971).

[8] S.O. Fatunla, Numerical Methods for Initial Value Problems in Ordinary Differential Equations, Academic Press, Boston (1988).

[9] L. Lustman B. Neta, W. Gragg, Solution of ordinary differential initial value problems on an intel hypercube, Computer Mathematics with Applications, 23 (1992), 65-72.

[10] H.B. Thompson, C. Tisdell, Systems of difference equations associated with boundary value problems for second order systems of ordinary differential equations, J. Math. Anal. Appl. 248 (2000) 333-347.

[11] Xiyon Cheng, Chengkui Zhong, Existence of positive solutions for a second order ordinary differential system, J. Math. Anal. Appl. 312 (2005) 14-23. 